formuliert, dass ein Bundeskanzler Mitglied des Bundestages sein müsse (S. 142), und auch die Behauptung, in deutschen Wahlkämpfen herrsche „de facto ein Zweiparteiensystem“, führt in die Irre (S. 143).

Die Stärke der Studie liegt jedoch in der konzisen Kurzdarstellung der Wahlkämpfe der vergangenen 50 Jahre in Deutschland und den USA, die sich auf umfassende Quellenarbeit stützt und entsprechende Verweise auf das fast 50-seitige Literaturverzeichnis beinhaltet. Überdies wurden zahlreiche Experteninterviews mit Politikberatern und wissenschaftlichen Beobachtern geführt, die in einer teilstandardisierten Form ausgewertet wurden.

Im Ergebnis hält Jochen W. Wagner fest, was in den vergangenen Jahren schon mehrfach formuliert wurde: Der Amerikanisierungs-Begriff ist den tatsächlichen Entwicklungen nicht angemessen. Viele der als neu wahrgenommenen Elemente moderner Wahlkampfführung (zum Beispiel Personalisierung, Zielgruppenansprache, Einsatz von Beratern, Nutzung von Demoskopie) sind nicht selten schon seit den 1950er Jahren in Deutschland etabliert. Ebenso lange beobachten die deutschen Parteizentralen intensiv amerikanische Wahlkämpfe, wobei keine blinde Übernahme von Techniken oder Strategien stattfindet: „Es zeigt sich vielmehr, dass eine eigenständige deutsche Kampagnenkultur besteht, die durch mehr als 50 Jahre Praxis so weit gefestigt ist, dass sie bedarfsorientiert und aus eigenem Antrieb selektiv Transferoptionen nutzen kann.“ (S. 405)

Die Zusammenschau der genannten Bücher macht deutlich: Wahlkämpfe entwickeln sich ausgesprochen dynamisch mit der verfügbaren Technologie, aber auch in Abhängigkeit von den politischen Rahmenbedingungen. Durchaus dienen dabei den Kandidaten, Parteien oder Medien auch internationale Erfahrungen als Inspiration. Bedingungen erfolgreicher Wahlkampfführung und die Diffusion von verschiedenen Innovationen in angemessener Komplexität zu erhellen, bleibt eine bedeutsame Herausforderung an Politik- und Kommunikationswissenschaft. Nur gut, dass das politische System Deutschlands auch weiterhin die Möglichkeit bietet, fast permanent Kampagnenforschung zu betreiben - denn irgendwo wird ja immer gewählt.

Sven T. Siefken

\title{
Standardwerk der qualitativen Nichtwählerforschung
}

Steinbrecher, Markus, Sandra Huber und Hans Rattinger: Turnout in Germany. Citizen Participation in State, Federal, and European Elections since 1979 (Studien zur Wahl-und Einstellungsforschung, Band 1), Nomos Verlagsgesellschaft, Baden-Baden 2007, 342 Seiten, € 49,-.

Nachdem sich der Fokus der deutschen Politikwissenschaft in den 1990er Jahren verstärkt auf die Wahlbeteiligung gerichtet hatte, begründet durch deren stetiges Absinken seit Ende der 1970er Jahre, wurde es erst einmal ruhiger auf diesem Gebiet. Der vorliegende Band ist daher eine längst überfällige Studie zur Erforschung von Wahlbeteiligung und individueller Wahlabstinenz in Deutschland. Sie zeichnet sich durch eine systematische und theoriegeleitete Vorgehensweise und die Nutzung umfangreichen empirischen Materials aus, das sowohl Bundestags-, Landtags- als auch Europawahlen berücksichtigt. Dadurch gelingt es den Autoren, den Stand der deutschen Nichtwählerforschung zu aktualisieren und zu erweitern. Ihr wesentliches Verdienst besteht in der akribischen Begehung nahezu aller bekannten Wege der Wahlforschung, um die Motive der Nichtwähler, aber auch die räumliche und zeitliche Struktur der Wahlbeteiligung zu ergründen. Lediglich der qualitative Pfad bleibt unbeschritten. 
„Turnout in Germany“ ist der erste Band der Serie „Studien zur Wahl- und Einstellungsforschung". Diese Positionierung verdeutlicht den zukünftigen Stellenwert der Nichtwählerforschung: Die Frage, ob die sinkende Wahlbeteiligung „Krise oder Normalisierung“ darstellt, ist von erheblicher Bedeutung für die Gesellschaft insgesamt. Folgerichtig wollen die Autoren erstens Gründe dafür ermitteln, warum die Wahlbereitschaft der Bürger nachlässt und zweitens erörtern, ob dies ein Problem offenbart.

Die Analyse ist in zwei Teile gegliedert, die sich an dem zur Verfügung stehenden Material orientieren. Zuerst werden Aggregat- und im Anschluss Individualdaten aus Befragungen ausgewertet. Die Autoren legen dar, dass sie aus der Analyse von Aggregatdaten keinen Beitrag zur Beantwortung ihrer zwei Kernfragen erwarten, da ihrer Ansicht nach das Wahlverhalten und damit auch die Nichtwahl von individuellen Einstellungen abhängt und somit nur Umfragedaten inhaltlich bedeutsame Ergebnisse liefern können. Umso höher ist ihnen anzurechnen, dass die Auswertung von Aggregatdaten genauso viel Platz einnimmt wie jene von Umfragedaten. Leider werden nur mechanisch eine Menge deskriptiver Kennzahlen ermittelt, ohne dass im Voraus Hypothesen aus den gängigen Theorien des Wahlverhaltens hergeleitet werden. An einigen Stellen treten Ungenauigkeiten auf, die zu Fehlurteilen führen. Zum Beispiel wird aus Standardabweichung und Range geschlossen, dass die Wahlbeteiligung im Osten Deutschlands bei Bundestagswahlen stabiler ist als im Westen. Abgesehen davon, dass diese Streuungsmaße bei trendbehafteten Zeitreihen wie der Wahlbeteiligung in dem betrachteten Zeitraum wenig sinnvoll sind, werden in Ost und West unterschiedliche Zeitintervalle betrachtet, wodurch auch die Interpretation von Mittelwerten schwierig wird. Dass die Wahlbeteiligung im Osten niedriger ist als im Westen, bleibt richtig, wird aber durch den Trend überschätzt. Ebenfalls fragwürdig ist die separate Berechnung von Regressionsmodellen zur Schätzung der Wahlbeteiligung in einzelnen Bundesländern, wodurch unterschiedliche länderspezifische Parameter geschätzt werden. Gerade der Einfluss der Sozialstruktur auf die Wahlbeteiligung sollte allgemein modelliert werden. Auf die Anwendung von Instrumenten aus der Zeitreihenanalyse wird verzichtet. Sie sind aber unentbehrlich, da der zu erklärenden Größe ein Trend unterstellt wird. Trotz dieser kleinen Makel, die im Übrigen den aktuellen Stand der Forschung widerspiegeln, wäre die Studie ohne die Auswertung der Aggregatdaten nur unvollständig. Besonders interessante Anknüpfungspunkte für die weitere Forschung liefern die Ergebnisse zum Einfluss von simultan stattfindenden Wahlen oder auch der Varianzzerlegung, die der Sozialstruktur auf der Aggregatebene nur einen sehr begrenzten Erklärungsgehalt der Varianz der Wahlbeteiligung zusprechen. Politische Konstellationen und Inhalte könnten bei künftigen Studien mehr Berücksichtigung finden.

In Kapitel 8 (,Why Do People Abstain?“), das sich der Analyse von Umfragen widmet, wird gezielt auf die gestellten Kernfragen eingegangen. Dieses und das folgende Kapitel sind besonders lesenswert, da in ihrem Verlauf die allgemeinen Theorien des Wahlverhaltens bezüglich der Nichtwahl mit dem vorhandenen Umfragematerial operationalisiert werden. Dabei werden unter anderem die Bedeutung der Wahlnorm, politisches Interesse, Einstellungen zum politischen System, Vertrauen in politische Akteure und Parteiendifferentiale thematisiert. Die Autoren finden Hinweise auf den Einfluss von Dealignment und Bestätigungen für Ansätze rationalen Wählens. Die Beteiligung an Bundestagswahlen wird, so Steinbrecher, Huber und Rattinger, stark durch Wahlnorm, Parteiidentifikation und politisches Interesse bestimmt. Ein interessanter Befund zur Beteiligung an Europawahlen ist der geringe Einfluss europaspezifischer und die große Bedeutung von allgemeinen Einstel- 
lungen zum politischen System. Aus methodischer Sicht sind die umfangreichen Ergebnisse, die hier im Einzelnen nicht ausgeführt werden können, aber nicht unproblematisch. Die Autoren verweisen auf das Problem der Unterschätzung des Nichtwähleranteils bei Umfragen durch systematische Falschangaben und Ausfälle. Sie bemerken sogar Zykluseffekte in Umfragen innerhalb der Wahlperioden, die nicht von der Erinnerungsleistung der Befragten abzuhängen scheinen. Darüber hinaus ist es äußerst fragwürdig, mit Regressionsparametern von Querschnittsdaten Trendentwicklungen erklären zu wollen.

Steinbrecher, Huber und Rattinger zeigen, dass die Erklärung der Wahlbeteiligung in ihren vielen Dimensionen nicht trivial ist. Die Bewertung der Ergebnisse - Krise oder Normalisierung - hängt letztlich vom normativen Standpunkt des Betrachters ab.

"Turnout in Germany“ ist eine Arbeit in der mehr als nur die Standardwerkzeuge der Wahlanalyse zum Einsatz kommen und wichtige Erkenntnisse um die Wahlbeteiligung und das individuelle Nichtwählen gewonnen und reproduziert werden. Das Buch spiegelt den aktuellen Stand der quantitativen Nichtwähler- und Wahlbeteiligungsforschung in Deutschland wider und wird auf absehbare Zeit das Standardwerk zu diesem Thema sein.

Ossip Fürnberg

\section{Gefüllte Lücke in der deutschen Wahl- und Einstellungsforschung}

Bytzek, Evelyn: Ereignisse und ihre Wirkung auf die Popularität von Regierungen. Von der Schleyer-Entführung zur Elbeflut (Studien zur Wahl-und Einstellungsforschung, Band 6), Nomos Verlagsgesellschaft, Baden-Baden 2007, 229 Seiten, € 34,90.

Evelyn Bytzek stellt sich in ihrer Dissertation dem Problem, wie politische Ereignisse auf die Popularität und damit auch die Wiederwahlchancen von Regierungen wirken. Dabei geht es zum einen um den bekannten Topos, dass Krisenzeiten Regierungszeiten wären. Das Elbehochwasser im Jahre 2002, das nach gängiger Interpretation Gerhard Schröder zur Wiederwahl verhalf, kann hier als paradigmatischer Fall gelten. Zum anderen stehen Skandale wie die CDU-Spendenaffäre 1999/2000, die Flick- und die Spiegelaffäre im Mittelpunkt sowie weitere Ereignistypen. Bytzek möchte herausfinden, welche Klassen von Ereignissen tatsächlich Popularität steigern, was genau die Wirkungsmechanismen sind sowie welcher Art und Dauer diese Wirkung ist. Ereignisse sollen also nicht ad-hoc analysiert werden, wie bislang üblich, wenn andere Erklärungen versagten, sondern systematisch und mit Blick auf regelmäßige Effekte.

Bei ihrem Vorhaben kann Bytzek an eine ausführlich in den USA geführte Debatte anknüpfen. Die dortige Situation könne, so die Autorin, jedoch nur in begrenztem Umfang auf die Bundesrepublik Deutschland übertragen werden. Zu unterschiedlich sei die Struktur der politischen Systeme und als Konsequenz daraus des Parteienwettbewerbs. Übertragbar ist jedoch die Feststellung, dass außerordentliche Ereignisse die Handlungsfähigkeit der Regierung fordern, Tätigkeitserwartungen erzeugen und Reaktionen der Regierung den Bürgern das Gefühl vermitteln müssen, bei den Amtsinhabern in allen Wechselfällen politischer Entwicklungen gut aufgehoben zu sein.

Um sich der Beantwortung ihrer Frage stringent zu nähern, definiert Bytzek zunächst den Begriff des Ereignisses (S. 49 ff.). Dieses hat drei Eigenschaften: (1) Es ist ein dem po- 